\title{
Simple Effective Control for Robot Manipulators with Friction
}

\author{
Maolin Jin, Sang Hoon Kang and Pyung Hun Chang \\ Korea Advanced Institute of Science and Technology (KAIST) \\ South Korea
}

\section{Introduction}

Friction accounts for more than $60 \%$ of the motor torque, and hard nonlinearities due to Coulomb friction and stiction severely degrade control performances as they account for nearly $30 \%$ of the industrial robot motor torque. Although many friction compensation methods are available (Armstrong-Helouvry et al., 1994; Lischinsky et al., 1999; Bona \& Indri, 2005;), here we only introduce relatively recent research works. Model-based friction compensation techniques (Mei et al., 2006; Bona et al., 2006; Liu et al., 2006) require prior experimental identification, and the drawback of these off-line friction estimation methods is that they can't adapt when the friction effects vary during the robot operations (Visioli et al., 2001). The adaptive compensation methods (Marton \& Lantos, 2007; Xie, 2006) take the modelling error of Lugre friction model into account, but they still require the complex prior experimental identification. Consequently, implementation of these schemes is highly complicated and computationally demanding due to the identification of many parameters and the calculation of the nonlinear friction model. Joint-torque sensory feedback (JTF) (Aghili \& Namvar, 2006) compensates friction without the dynamic models, but high price torque sensors are needed for the implementation of JTF.

To give satisfactory solution to robot control, in general, we should continue our research in modelling the robot dynamics and nonlinear friction so that they become applicable to wider problem domain. In the mean time, when the parameters of robot dynamics are poorly understood, and for the ease of practical implementation, simple and efficient techniques may represent a viable alternative.

Accordingly, the authors developed a non-model based control technique for robot manipulators with nonlinear friction (Jin et al., 2006; Jin et al., 2008). The nonlinear terms in robot dynamics are classified into two categories from the time-delay estimation (TDE) viewpoint: soft nonlinearities (gravity, viscous friction, and Coriolis and centrifugal torques, disturbances, and interaction torques) and hard nonlinearities (due to Coulomb friction, stiction, and inertia force uncertainties). TDE is used to cancel soft nonlinearities, and ideal velocity feedback (IVF) is used to suppress the effect of hard nonlinearities. We refer the control technique as time-delay control with ideal velocity feedback (TDCIVF). Calculation of complex robot model and nonlinear friction model is not required in the TDCIVF. The TDCIVF control structure is transparent to designers; it consists of three elements that have clear meaning: a soft nonlinearity cancelling element, a hard nonlinearity suppressing 
element, and a target dynamics injecting element. The TDCIVF turns out simple in form and easy to tune; specifying desired dynamics for robots is all that is necessary for a user to do. The same control structure can be used for both free space motion and constrained motion of robot manipulators.

This chapter is organized as follows. After briefly review TDCIVF (Jin et al., 2006; Jin et al., 2008), the implementation procedure and discussion are given in section 2 . In section 3 , simulations are carried out to examine the cancellation effect of soft nonlinearities using TDE and the suppression effect of hard nonlinearities using IVF. In section 4, the TDCIVF is compared with adaptive friction compensation (AFC) (Visioli et al., 2001; Visioli et al., 2006; Jatta et al., 2006), a non-model based friction compensation method that is similar to the TDCIVF in that it requires neither friction identification nor expensive torque sensor. Cartesian space formulation is presented in section 5. An application of human-robot cooperation is presented using a two-degree-of-freedom (2-DOF) SCARA-type industrial robot in section 6. Finally, section 7 concludes the paper.

\section{Time-Delay Control with Ideal Velocity Feedback}

\subsection{Classification of Robot Dynamics from TDE Viewpoint}

The dynamics equation of $\mathrm{n}-\mathrm{DOF}$ robot manipulator in joint space coordinates is given by:

$$
\tau=M(\theta) \ddot{\theta}+V(\theta, \dot{\theta})+G(\theta)+F+D-\tau_{s}
$$

where $\tau \in \mathfrak{R}^{n}$ denotes actuator torque and $\tau_{s} \in \mathfrak{R}^{n}$ interaction torque; $\boldsymbol{\theta}, \dot{\boldsymbol{\theta}}, \ddot{\boldsymbol{\theta}} \in \mathfrak{R}^{n}$ denote the joint angle, the joint velocity, and the joint acceleration, respectively; $\mathbf{M}(\boldsymbol{\theta}) \in \mathfrak{R}^{n \times n}$ a positive definite inertia matrix; and $\mathbf{V}(\boldsymbol{\theta}, \dot{\boldsymbol{\theta}}) \in \mathfrak{R}^{n}$ Coriolis and centrifugal torque; $\mathbf{G}(\boldsymbol{\theta}) \in \mathfrak{R}^{n}$ a gravitational force; $\mathbf{F} \in \mathfrak{R}^{n}$ stands for the friction term including Coulomb friction, viscous friction and stiction; and $\mathbf{D} \in \mathfrak{R}^{n}$ continuous disturbance.

Introducing a constant matrix $\overline{\mathbf{M}} \in \Re^{n \times n}$, one can obtain another expression of (1) as follows:

$$
\tau=\overline{\mathbf{M}} \ddot{\theta}+\mathbf{N}(\boldsymbol{\theta}, \dot{\theta}, \ddot{\theta})
$$

where $\mathbf{N}(\boldsymbol{\theta}, \dot{\boldsymbol{\theta}}, \ddot{\boldsymbol{\theta}})$ includes all the nonlinear terms of robot dynamics as

$$
\mathbf{N}(\boldsymbol{\theta}, \dot{\boldsymbol{\theta}}, \ddot{\boldsymbol{\theta}})=[\mathbf{M}(\boldsymbol{\theta})-\overline{\mathbf{M}}] \ddot{\theta}+\mathrm{V}(\boldsymbol{\theta}, \dot{\boldsymbol{\theta}})+\mathrm{G}(\boldsymbol{\theta})+\mathrm{F}+\mathrm{D}-\mathbf{\tau}_{s}
$$

$\mathbf{N}(\boldsymbol{\theta}, \dot{\boldsymbol{\theta}}, \ddot{\boldsymbol{\theta}})$ can be classified into two categories as follows (Jin et al., 2008):

$$
\begin{gathered}
\mathbf{N}(\boldsymbol{\theta}, \dot{\boldsymbol{\theta}}, \ddot{\boldsymbol{\theta}})=\mathbf{S}(\boldsymbol{\theta}, \dot{\boldsymbol{\theta}})+\mathbf{H}(\boldsymbol{\theta}, \dot{\boldsymbol{\theta}}, \ddot{\boldsymbol{\theta}}), \\
\mathbf{S}(\boldsymbol{\theta}, \dot{\boldsymbol{\theta}})=\mathbf{V}(\boldsymbol{\theta}, \dot{\boldsymbol{\theta}})+\mathbf{G}(\boldsymbol{\theta})+\mathbf{F}_{v}(\dot{\boldsymbol{\theta}})+\mathbf{D}-\mathbf{\tau}_{s} \\
\mathbf{H}(\boldsymbol{\theta}, \dot{\boldsymbol{\theta}}, \ddot{\boldsymbol{\theta}})=\mathbf{F}_{c}(\dot{\boldsymbol{\theta}})+\mathrm{F}_{s t}(\boldsymbol{\theta}, \dot{\boldsymbol{\theta}})+[\mathbf{M}(\boldsymbol{\theta})-\overline{\mathbf{M}}] \ddot{\boldsymbol{\theta}},
\end{gathered}
$$


where $\mathbf{F}_{v}, \mathbf{F}_{c}, \mathbf{F}_{s t} \in \mathfrak{R}^{n}$ denote viscous friction, Coulomb friction, stiction, respectively. When the time-delay $L$ is sufficiently small, it is very reasonable to assume that $\mathbf{S}(\boldsymbol{\theta}, \dot{\boldsymbol{\theta}})$ is closely approximated by $\mathbf{S}(\boldsymbol{\theta}, \dot{\boldsymbol{\theta}})_{t-L}$ shwon in Fig. 1. It is expressed by

$$
\hat{\mathbf{S}}(\boldsymbol{\theta}, \dot{\boldsymbol{\theta}})=\mathbf{S}(\boldsymbol{\theta}, \dot{\boldsymbol{\theta}})_{t-L} \cong \mathbf{S}(\boldsymbol{\theta}, \dot{\boldsymbol{\theta}}) .
$$

$\operatorname{But} \mathbf{H}(\boldsymbol{\theta}, \dot{\boldsymbol{\theta}}, \ddot{\boldsymbol{\theta}})$ is not compatible with the TDE technique, as

$$
\hat{\mathbf{H}}(\boldsymbol{\theta}, \dot{\boldsymbol{\theta}}, \ddot{\theta})=\mathbf{H}(\boldsymbol{\theta}, \dot{\theta}, \ddot{\theta})_{t-L} \neq \mathbf{H}(\boldsymbol{\theta}, \dot{\theta}, \ddot{\theta}) .
$$

In this paper, $\hat{\bullet}$ denotes estimated value of $\bullet$, and $\bullet_{t-L}$ denotes time delayed value of $\bullet$. Incidentally, time-delay estimation (TDE) is originated from pioneering works called timedelay control (TDC) (Youcef-Toumi \& Ito, 1990; Hsia et al., 1991). However, little attention has been paid to hard nonlinearities such as Coulomb friction and stiction forces in previous researches on TDC.

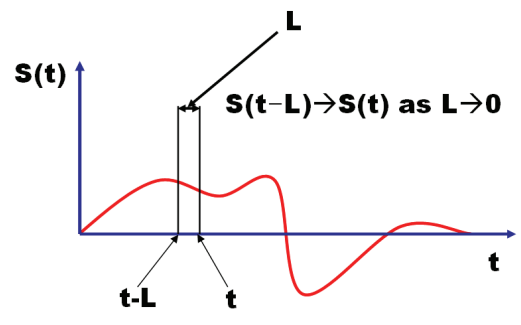

(a) TDE of soft nonlinearities

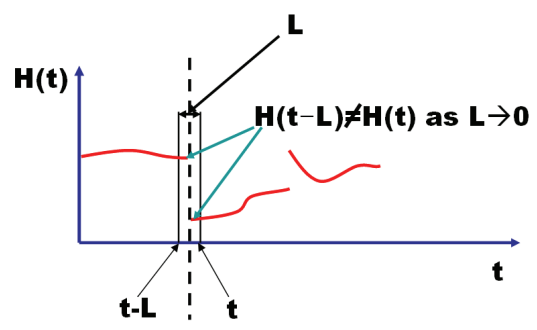

(b) TDE of hard nonlinearities

Figure 1. Description of TDE of soft and hard nonlinearities

\subsection{Target Dynamics}

The control objective is to make the robot to achieve the following target impedance dynamics:

$$
\mathbf{M}_{d}\left(\ddot{\boldsymbol{\theta}}_{d}-\ddot{\boldsymbol{\theta}}\right)+\mathbf{B}_{d}\left(\dot{\boldsymbol{\theta}}_{d}-\dot{\boldsymbol{\theta}}\right)+\mathbf{K}_{d}\left(\boldsymbol{\theta}_{d}-\boldsymbol{\theta}\right)+\mathbf{\tau}_{s}=\mathbf{0},
$$

where $\mathbf{M}_{d} \in \mathfrak{R}^{n \times n}, \mathbf{K}_{d} \in \mathfrak{R}^{n \times n}, \quad \mathbf{B}_{d} \in \mathfrak{R}^{n \times n}$, denote desired mass, spring, and damper, respectively; $\boldsymbol{\theta}_{d}, \dot{\boldsymbol{\theta}}_{d}, \ddot{\boldsymbol{\theta}}_{d} \in \mathfrak{R}^{n}$ the desired position, desired velocity, desired acceleration, respectively. When the robot is performing free space motion, the sensed torque $\boldsymbol{\tau}_{s}$ is 0 , and (9) is reduced to the well-known error dynamics of motion control; it is expressed by

$$
\ddot{\boldsymbol{\theta}}_{d}-\ddot{\boldsymbol{\theta}}+\mathbf{K}_{V}\left(\dot{\boldsymbol{\theta}}_{d}-\dot{\boldsymbol{\theta}}\right)+\mathbf{K}_{P}\left(\boldsymbol{\theta}_{d}-\boldsymbol{\theta}\right)=\mathbf{0}
$$

with

$$
\mathbf{K}_{V} \triangleq \mathbf{M}_{d}^{-1} \mathbf{B}_{d}, \quad \mathbf{K}_{p} \triangleq \mathbf{M}_{d}^{-1} \mathbf{K}_{d}
$$




\subsection{Actual Dynamics When Only TDE is Used}

The robot dynamics equation can be rewritten (2) as

$$
\tau=\overline{\mathbf{M}} \ddot{\theta}+S(\boldsymbol{\theta}, \dot{\theta})+\mathbf{H}(\boldsymbol{\theta}, \dot{\theta}, \ddot{\theta}) .
$$

The control input using TDE is

$$
\tau=\overline{\mathbf{M}} \mathbf{u}+\hat{S}(\boldsymbol{\theta}, \dot{\theta})+\hat{\mathbf{H}}(\boldsymbol{\theta}, \dot{\boldsymbol{\theta}}, \ddot{\theta}) .
$$

where

$$
\mathbf{u}=\ddot{\boldsymbol{\theta}}_{d}+\mathbf{M}_{d}^{-1}\left[\mathbf{K}_{d}\left(\boldsymbol{\theta}_{d}-\boldsymbol{\theta}\right)+\mathbf{B}_{d}\left(\dot{\boldsymbol{\theta}}_{d}-\dot{\boldsymbol{\theta}}\right)+\mathbf{\tau}_{s}\right]
$$

In (13), $\hat{\mathbf{S}}(\boldsymbol{\theta}, \dot{\boldsymbol{\theta}})+\hat{\mathbf{H}}(\boldsymbol{\theta}, \dot{\boldsymbol{\theta}}, \ddot{\boldsymbol{\theta}})$ is obtained from the TDE of $\mathbf{S}(\boldsymbol{\theta}, \dot{\boldsymbol{\theta}})+\mathbf{H}(\boldsymbol{\theta}, \dot{\boldsymbol{\theta}}, \ddot{\boldsymbol{\theta}})$, expressed by

$$
\hat{\mathbf{S}}(\boldsymbol{\theta}, \dot{\boldsymbol{\theta}})+\hat{\mathbf{H}}(\boldsymbol{\theta}, \dot{\boldsymbol{\theta}}, \ddot{\boldsymbol{\theta}})=\mathbf{S}(\boldsymbol{\theta}, \dot{\boldsymbol{\theta}})_{t-L}+\mathbf{H}(\boldsymbol{\theta}, \dot{\boldsymbol{\theta}}, \ddot{\boldsymbol{\theta}})_{t-L}=\mathbf{\tau}_{t-L}-\overline{\mathbf{M}} \ddot{\boldsymbol{\theta}}_{t-L} .
$$

With the combination of (7),(12)-(15), and (17), one can obtain actual impedance error dynamics as

$$
\mathbf{M}_{d}\left(\ddot{\boldsymbol{\theta}}_{d}-\ddot{\boldsymbol{\theta}}\right)+\mathbf{B}_{d}\left(\dot{\boldsymbol{\theta}}_{d}-\dot{\boldsymbol{\theta}}\right)+\mathbf{K}_{d}\left(\boldsymbol{\theta}_{d}-\boldsymbol{\theta}\right)+\mathbf{\tau}_{s}=\mathbf{M}_{d} \varepsilon .
$$

where the TDE error $\varepsilon$ is defined as

$$
\varepsilon \triangleq \overline{\mathbf{M}}^{-1}\left[\mathbf{H}(\boldsymbol{\theta}, \dot{\boldsymbol{\theta}}, \ddot{\boldsymbol{\theta}})-\mathbf{H}(\boldsymbol{\theta}, \dot{\boldsymbol{\theta}}, \ddot{\boldsymbol{\theta}})_{t-L}\right] .
$$

\subsection{Hard Nonlinearity Compensation}

TDCIVF uses ideal velocity feedback (IVF) term in order to suppress $\varepsilon$ that causes the deviation of resulting dynamics from the target impedance dynamics, as

$$
\mathbf{\tau}=\mathbf{\tau}_{t-L}-\overline{\mathbf{M}} \ddot{\boldsymbol{\theta}}_{t-L}+\overline{\mathbf{M}}\left\{\ddot{\boldsymbol{\theta}}_{d}+\mathbf{M}_{d}^{-1}\left[\mathbf{\tau}_{s}+\mathbf{K}_{d}\left(\boldsymbol{\theta}_{d}-\boldsymbol{\theta}\right)+\mathbf{B}_{d}\left(\dot{\boldsymbol{\theta}}_{d}-\dot{\boldsymbol{\theta}}\right)\right]+\boldsymbol{\Gamma}\left(\dot{\boldsymbol{\theta}}_{\text {ideal }}-\dot{\boldsymbol{\theta}}\right)\right\}
$$

where $\Gamma \in \Re^{n \times n}$ denotes a positive definite diagonal matrix, and

$$
\dot{\boldsymbol{\theta}}_{\text {ideal }}=\int\left\{\ddot{\boldsymbol{\theta}}_{d}+\mathbf{M}_{d}^{-1}\left[\mathbf{K}_{d}\left(\boldsymbol{\theta}_{d}-\boldsymbol{\theta}\right)+\mathbf{B}_{d}\left(\dot{\boldsymbol{\theta}}_{d}-\dot{\boldsymbol{\theta}}\right)+\mathbf{\tau}_{s}\right]\right\} d t .
$$

If we considere the integral sliding surface as

$$
\mathbf{s}=\int\left\{\ddot{\boldsymbol{\theta}}_{d}-\ddot{\boldsymbol{\theta}}+\mathbf{M}_{d}^{-1}\left[\mathbf{\tau}_{s}+\mathbf{K}_{d}\left(\boldsymbol{\theta}_{d}-\boldsymbol{\theta}\right)+\mathbf{B}_{d}\left(\dot{\boldsymbol{\theta}}_{d}-\dot{\boldsymbol{\theta}}\right)\right]\right\} d t .
$$

Then, (19) reduces to $\mathbf{s}=\dot{\boldsymbol{\theta}}_{\text {ideal }}-\dot{\boldsymbol{\theta}}$, and (18) can be expressed by

$$
\mathbf{\tau}=\mathbf{\tau}_{t-L}-\overline{\mathbf{M}} \ddot{\boldsymbol{\theta}}_{t-L}+\overline{\mathbf{M}}\left\{\ddot{\boldsymbol{\theta}}_{d}+\mathbf{M}_{d}^{-1}\left[\mathbf{\tau}_{s}+\mathbf{K}_{d}\left(\boldsymbol{\theta}_{d}-\boldsymbol{\theta}\right)+\mathbf{B}_{d}\left(\dot{\boldsymbol{\theta}}_{d}-\dot{\boldsymbol{\theta}}\right)\right]+\Gamma \mathbf{s}\right\} .
$$

The s-trajectory, which is integral error of target dynamics, represents a time-varying measure of impedance error (Slotine, 1985). According to Barbalat's Lemma, as the sliding surface $s(t) \rightarrow 0$, we can expect that $\dot{\mathbf{s}}(t) \rightarrow 0$, which implies achieving desired impedance (9) as time $\rightarrow \infty$. When the robot is performing free space motion, the sensed torque/force, $\mathbf{\tau}_{s}$ is 0 ; $\dot{\boldsymbol{\theta}}_{\text {ideal }}-\dot{\boldsymbol{\theta}} \rightarrow 0$ implies $\boldsymbol{\theta} \rightarrow \boldsymbol{\theta}_{d}$.

Stability analysis can be found in (Jin et al., 2008). 


\subsection{The Simple, Transparent Control Structure}

The TDCIVF has transparent structure as shown in (22). It consists of three elements that have clear meaning: a soft nonlinearity cancelling element, a hard nonlinearity suppressing element, and a target dynamics injecting element.

$$
\begin{aligned}
\tau=\underbrace{\tau_{t-L}-\overline{\mathbf{M}} \ddot{\theta}_{t-L}}_{\text {cancelling soft nonlinearities }}+\underbrace{\overline{\mathbf{M}} \ddot{\theta}_{d}+\mathbf{M}_{d}^{-1}\left[\mathbf{\tau}_{s}+\mathbf{K}_{d}\left(\boldsymbol{\theta}_{d}-\boldsymbol{\theta}\right)+\mathbf{B}_{d}\left(\dot{\boldsymbol{\theta}}_{d}-\dot{\boldsymbol{\theta}}\right)\right]}_{\text {injecting target impedance dynamics }}\} \\
+\underbrace{\overline{\mathbf{M}} \Gamma\left(\dot{\boldsymbol{\theta}}_{\text {ideal }}-\dot{\boldsymbol{\theta}}\right)}_{\text {suppressing the effect of hard nonlinearities }} .
\end{aligned}
$$

Among the nonlinear terms in the robot dynamics, soft nonlinearities (gravity, viscous friction, and Coriolis and centrifugal torques, disturbances, and interaction torques) are cancelled by TDE, $\boldsymbol{\tau}_{t-L}-\overline{\mathbf{M}} \ddot{\boldsymbol{\theta}}_{t-L}$; and hard nonlinearities (due to Coulomb friction, stiction, and inertia force uncertainties) are suppressed by IVF $\boldsymbol{\Gamma} \cdot\left(\dot{\boldsymbol{\theta}}_{\text {ideal }}-\dot{\boldsymbol{\theta}}\right)$; thus, calculations of complex robot dynamics as well as that of nonlinear friction are unnecessary, and the controller is easy to implement. Only two gain matrices, $\overline{\mathbf{M}}$ and $\boldsymbol{\Gamma}$, must be tuned for the control law. The gains have clear meaning: $\overline{\mathbf{M}}$ is for soft nonlinearities cancellation and noise attenuation, and $\Gamma$ is for hard nonlinearities suppression. Consequently, the TDCIVF turns out simple in form and easy to tune; specifying the target dynamics for robot is all that is necessary for a user to do.

\subsection{Implementation Procedure}

The implementation procedure is straightforward as follows:

1. Describe desired trajectory $\boldsymbol{\theta}_{d}$ according to task requirements.

2. Select $\mathbf{K}_{d}$ and $\mathbf{M}_{d}$ according to task requirements and the approximate value of robot inertia.

3. Select $\mathbf{B}_{d}$ by applying critically damped condition .

4. Set $\Gamma=0$ and tune $\overline{\mathbf{M}}$. Start with a small positive value, and increase it. Stop increasing $\overline{\mathbf{M}}$ when robot joints become noisy. The elements of $\overline{\mathbf{M}}$ can be tuned separately.

5. Tune $\Gamma$. Increase it from zero to an appropriate value. The elements of $\Gamma$ can be tuned separately.

Incidentally, the digital implementation of TDCIVF is as follows:

1. Following numerical integration is used to calculate ideal velocity:

$$
\dot{\boldsymbol{\theta}}_{\text {ideel }}(t)=\dot{\boldsymbol{\theta}}_{\text {ideal }}(t-L)+L\left\{\ddot{\boldsymbol{\theta}}_{d}+\mathbf{M}_{d}^{-1}\left[\mathbf{K}_{d}\left(\boldsymbol{\theta}_{d}-\boldsymbol{\theta}\right)+\mathbf{B}_{d}\left(\dot{\boldsymbol{\theta}}_{d}-\dot{\boldsymbol{\theta}}\right)+\mathbf{\tau}_{s}\right]\right\} .
$$

2. $\ddot{\boldsymbol{\theta}}_{t-L}$ and $\dot{\boldsymbol{\theta}}$ are calculated by numerical differentiation (24) to implement the TDCIVF.

$$
\ddot{\boldsymbol{\theta}}_{t-L}=\left(\dot{\boldsymbol{\theta}}_{t}-\dot{\boldsymbol{\theta}}_{t-L}\right) / L \text { and } \dot{\boldsymbol{\theta}}=\left(\boldsymbol{\theta}_{t}-\boldsymbol{\theta}_{t-L}\right) / L \text {. }
$$




\subsection{Drawbacks}

The drawback of TDCIVF inherits from TDE. TDE term $\mathbf{\tau}_{t-L}-\overline{\mathbf{M}} \ddot{\boldsymbol{\theta}}_{t-L}$ in (22) needs numerical differentiations (24) which may amplify the effect of noise and deteriorate the control performance. Thus, the elements of $\overline{\mathbf{M}}$ are lowered for practical use to filter the noise (Jin et al., 2008).

\section{Simulation Studies}

Simulations are carried out to examine the cancellation effect of soft nonlinearities using TDE and the suppression effect of hard nonlinearities using IVF. Viscous friction parameters (soft nonlinearities), and Coulomb friction parameters (hard nonlinearities) are varied while other parameters remain constant in simulations to see how TDE affects the compensation of nonlinear terms.

If $\Gamma=0$ in the TDCIVF (22), we can obtain the formulation as follows:

$$
\tau=\tau_{t-L}-\bar{M} \ddot{\theta}_{t-L}+\bar{M}\left\{\ddot{\theta}_{d}+M_{d}^{-1}\left[\tau_{s}+K_{d}\left(\theta_{d}-\theta\right)+B_{d}\left(\dot{\theta}_{d}-\dot{\theta}\right)\right]\right\} .
$$

This formulation is referred to as internal force based impedance control (IFBIC) in (Bonitz \& Hsia, 1996; Lasky \& Hsia, 1991), where the compensation of the hard nonlinearities was not considered.

For simplicity and clarity, a single arm with soft and hard nonlinearities is considered as shown in Fig. 2. The simulation parameters are as follows: The mass of the link is $m=8.163 \mathrm{Kg}$, the link length is $l=0.35 \mathrm{~m}$, and the inertia is $I=1.0 \mathrm{Kgm}^{2}$; the stiffness of the external spring as disturbance is $K_{\text {disturbance }}=10 \mathrm{Nm} / \mathrm{rad}$; the acceleration due to gravity is $g=9.8 \mathrm{Kgm} / \mathrm{s}^{2}$. The parameters of environment are $K_{e}=12000 \mathrm{Nm} / \mathrm{rad}$ and $B_{e}=0.40 \mathrm{Nms} / \mathrm{rad}$; its location is at $\theta_{e}=0.2 \mathrm{rad}$. The sampling frequency of the simulation is $1 \mathrm{KHz}$.

The dynamics of a single arm is

$$
\tau=I \ddot{\theta}+G(\theta)+F_{v}(\dot{\theta})+F_{c}(\dot{\theta})+d
$$

where

$$
\begin{gathered}
G(\theta)=m g l \sin (\theta) \\
d=-K_{\text {disturbance }} \theta \\
F_{v}(\dot{\theta})=-C_{v} \dot{\theta} \\
F_{c}(\dot{\theta})=-C_{c} \operatorname{sgn}(\dot{\theta}) .
\end{gathered}
$$

Soft nonlinearities and hard nonlinearities can be expressed by

$$
\begin{gathered}
S(\theta, \dot{\theta})=G(\theta)+F_{v}(\dot{\theta})+d, \\
H(\theta, \dot{\theta}, \ddot{\theta})=F_{c}(\dot{\theta}) .
\end{gathered}
$$

For comparisons, identical parameters are used for the target dynamics in free space motion, as follows: $M_{d}=1.0 \mathrm{Kgm}^{2}, K_{d}=100 \mathrm{Nm} / \mathrm{rad}$, and $B_{d}=20 \mathrm{Nms} / \mathrm{rad}$.

The desired trajectory is given by (33)

$$
\theta_{d}=A[1-\exp (-\omega \mathrm{t})] \sin (\omega t)
$$


where $\omega=2 \pi / p, p=5 \mathrm{~s}, A=0.15 \mathrm{rad}$.

The simulation results are arranged in Figs. 3-7. The tracking errors of IFBIC with fixed Coulomb friction coefficients and various viscous friction coefficients are shown in Fig. 4 (a). The viscous friction has little effect on the tracking error because it can be cancelled by TDE. The tracking errors of IFBIC with various Coulomb friction coefficients and fixed viscous friction coefficients are shown in Fig. 4 (b). The larger Coulomb friction results in the larger tracking error. These results imply that TDE can not cancel hard nonlinearities. Maximum absolute errors and Mean absolute errors show that Coulomb friction severely deteriorates tracking errors whereas viscous friction has little effect on tracking errors shown in Figs. 4 (c) and (d).

Fig. 5 (a) shows soft nonlinearities $S(\theta, \dot{\theta})$ and the TDE error of soft nonlinearities $S(\theta, \dot{\theta})-S(\theta, \dot{\theta})_{t-L}$. Because soft nonlinearities are continuous functions, the TDE error of soft nonlinearities is almost zero, and TDE works well on the cancellation of soft nonlinearities. Fig. 5 (b) shows hard nonlinearities $H(\theta, \dot{\theta}, \ddot{\theta})$ and the TDE error of hard nonlinearities $H(\theta, \dot{\theta}, \ddot{\theta})-H(\theta, \dot{\theta}, \ddot{\theta})_{t-L}$. Because hard nonlinearities are discontinuous functions, the TDE error of hard nonlinearities cannot be ignored. The TDE error of Coulomb friction can be regarded as a pulse type disturbance when the velocity changes its sign. The TDCIVF can reduce the tracking error due to Coulomb friction by increasing $\Gamma$, as shown in Fig. 6 .

The element of control input of the TDCIVF due to IVF term, $\overline{\mathbf{M}} \Gamma\left(\dot{\boldsymbol{\theta}}_{\text {ideal }}-\dot{\boldsymbol{\theta}}\right)$, is plotted in Fig. 7. The IVF term is almost zero in the presence of only soft nonlinearities in Figs. 7 (a) and (b); however, it activates in the presence of hard nonlinearities Figs. 7 (c) and (d).

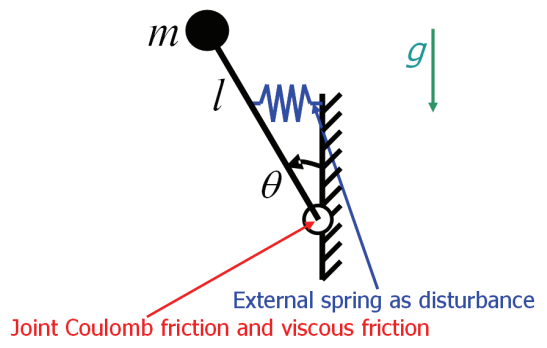

Figure 2. A single arm with friction
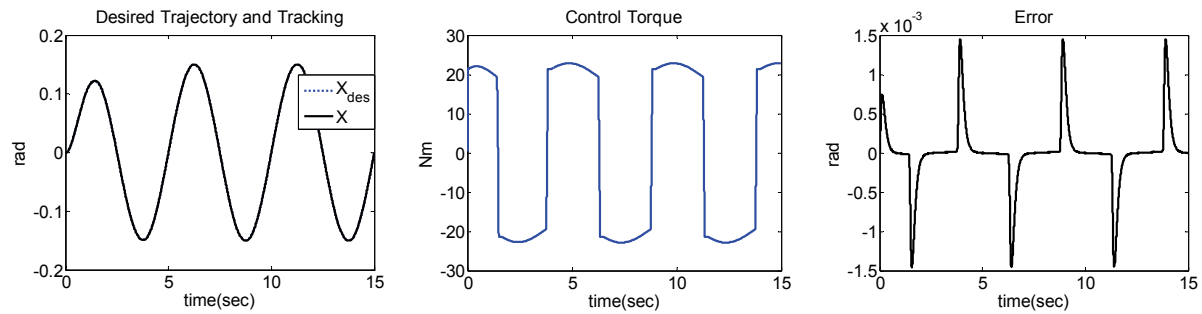

Figure 3. Simulation results with Coulomb friction coefficient $C_{c}=20$ and viscous friction coefficient $C_{v}=15$ 

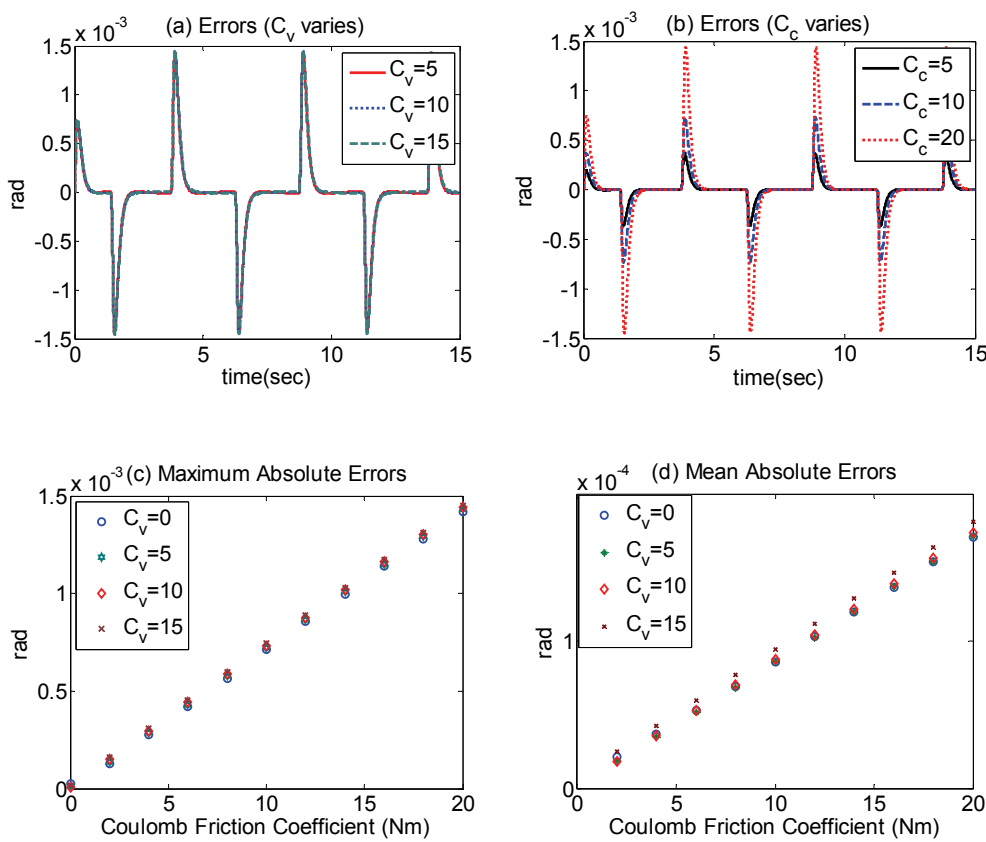

Figure 4. Tracking errors of IFBIC: (a) with fixed Coulomb friction $\left(C_{c}=20\right)$ and various $C_{v}$. (b) with fixed viscous friction $\left(C_{v}=10\right)$ and various $C_{c}$. (c) and (d) Maximum (Mean) absolute errors with various $C_{c}$ and $C_{v}$. It shows that Coulomb friction severely deteriorates the tracking performance, and viscous friction has little effect on tracking errors

(a) Soft Nonlinearity \& Estimation Error

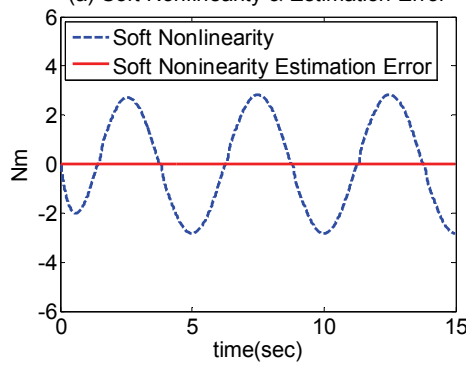

(b) Hard Nonlinearity \& Estimation Error

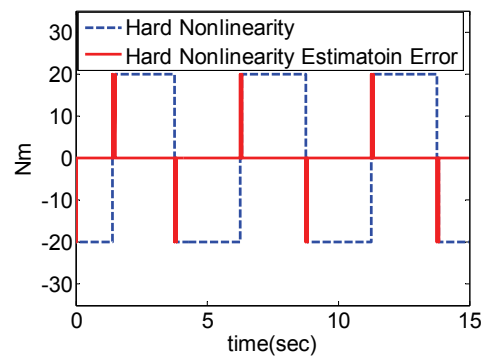

Figure 5. Nonlinear terms and their estimation errors. (a) Soft nonlinearity and its estimation error. (b) Hard nonlinearity and its estimation error. $\left(C_{c}=20, C_{v}=15\right.$.) 

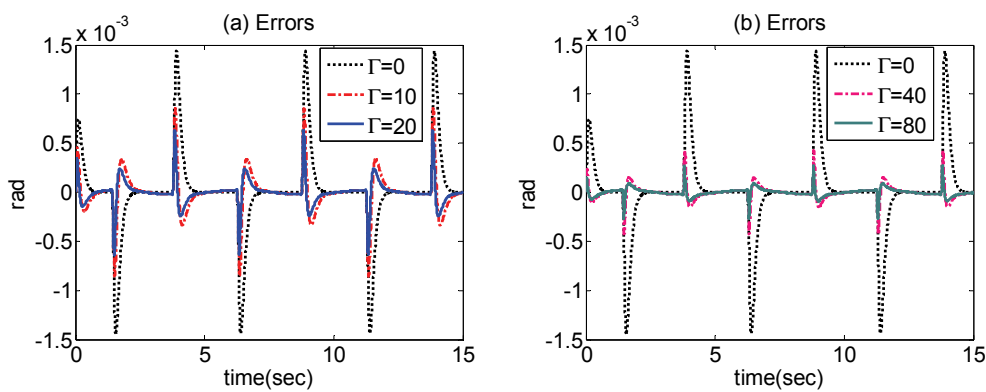

Figure 6. The effect of gain $\Gamma$ in $\operatorname{TDCIVF}\left(C_{c}=20, C_{v}=10\right)$. (a) $\Gamma=0,10,20$; (b) $\Gamma=0,40,80$
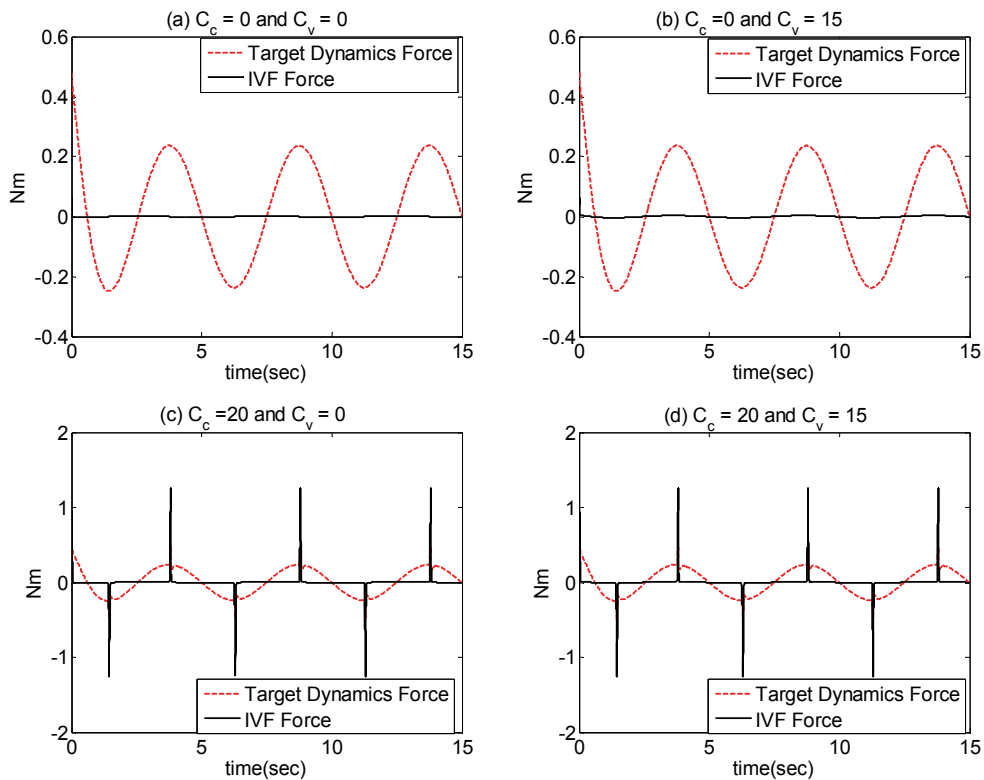

Figure 7. TDCIVF control input: Target dynamics injection torque and ideal velocity feedback torque. The IVF does not activate when hard nonlinearity is $0\left(C_{c}=0\right)$ in (a) and (b); The IVF activates when hard nonlinearities do exist $\left(C_{c}=20\right)$ in (c) and (d)

\section{Comparison with Adaptive Friction Compensation Method}

Adaptive friction compensation (Visioli et al., 2001; Visioli et al., 2006; Jatta et al., 2006) is regarded as a promising non-model based technique and it provides simple, effective online friction compensation. Hence, the TDCIVF is compared with AFC, a recently developed friction compensation method. 


\subsection{Adaptive Friction Compensation}

First we briefly review the AFC (Visioli et al., 2001; Visioli et al., 2006; Jatta et al., 2006). As shown in Fig. 11, the AFC algorithm assumes that the difference between torque estimation error $u_{i}^{t}$ and its estimation $u_{i}$ depends only on the friction for each joint $i$. Thus, the estimation error between the actual friction torque $f_{i}^{a}\left(\dot{q}_{i}\right)$ and estimated one $f_{i}\left(\dot{q}_{i}\right)$ can be approximately considered equal to the output of the PID controller, i.e.:

$$
f_{i}^{a}\left(\dot{q}_{i}\right)-f_{i}\left(\dot{q}_{i}\right) \cong u_{i}^{r}
$$

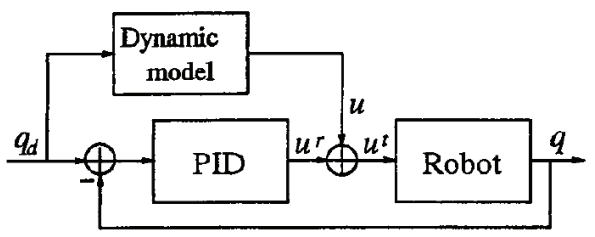

Figure 11. The general model for adaptive friction compensation

The friction terms are approximated by polynomial functions of degree $h$. Positive and negative velocities might be considered separately to obtain better results in case the actual friction functions is not symmetrical; hence,

$$
f_{i}\left(\dot{q}_{i}\right)=\left\{\begin{array}{ll}
p_{i 0}^{-}+p_{i 1}^{-} \dot{q}_{i}+\cdots+p_{i h}^{-} \dot{q}_{i}^{h} & \text { if } \dot{q}_{i}<0 \\
p_{i 0}^{+}+p_{i 1}^{+} \dot{q}_{i}+\cdots+p_{i h}^{+} \dot{q}_{i}^{h} & \text { if } \dot{q}_{i}>0
\end{array}(i=1, \cdots, n) .\right.
$$

Formally, the method can be described as follows. Define

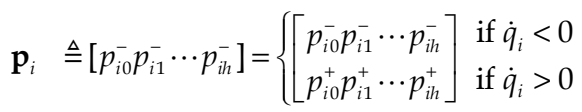

and

$$
\mathbf{v}_{i} \triangleq\left[\begin{array}{llll}
1 & \dot{q}_{i} & \cdots & \dot{q}_{i}^{h}
\end{array}\right]
$$

The algorithm can be described as follows:

a. For $(i=1, \cdots, n)$

1. Measure $q_{i}(k)$ and $\dot{q}_{i}(k)$.

2. Calculate $\ddot{q}_{i}(k)$ by differentiation and filtering.

3. Set $e_{i}(k)=u_{i}^{r}(k)$.

4. Calculate $\Delta \mathbf{p}_{i}(k)=\eta e_{i}(k) \mathbf{v}_{i}(k)+\alpha \Delta \mathbf{p}_{i}(k-1)$.

5. Set $\mathbf{p}_{i}(k)=\mathbf{p}_{i}(k-1)+\Delta \mathbf{p}_{i}(k)$.

6. Calculate $u_{i}^{t}=u_{i}+u_{i}^{r}$.

b. Apply the reference command torque signal $u_{i}^{t}$. The parameter $\eta$ determines the velocity of the descent to the minimum and therefore the adaptation velocity. 
Parameter $\alpha$ is the momentum coefficient, which helps to prevent large oscillations of the weight values and to accelerate the descent when the gradient is small.

\subsection{Comparisons}

For simplicity and clarity, we present experimental results using a single arm robot with friction. As shown in Fig. 12 (a), a single arm robot is commanded to move very fast in free space repeatedly $(\mathrm{t}=0-6 \mathrm{~s})$. A $5^{\text {th }}$ polynomial desired trajectory is used for 8 path segments listed in Table 1, where both the initial position and the final position of each segment are listed along with the initial time and the final time. The velocity and the acceleration at the beginning and end of each path segment are set to zero.

\begin{tabular}{|l|r|r|r|r|r|r|r|r|l|}
\hline TIME(S) & 0.0 & 0.5 & 1.5 & 2.5 & 3.5 & 4.5 & 5.5 & 6.0 & 7.0 \\
\hline X(RAD) & 0.0 & 0.1 & -0.1 & 0.1 & -0.1 & 0.1 & -0.1 & 0.0 & 0.0 \\
\hline
\end{tabular}

Table 1. The desired trajectory

To implement AFC, the method described in previous section was followed exactly.

The PID control is expressed as

$$
u(t)=K\left(e(t)+T_{D} \dot{e}(t)+T_{I}^{-1} \int_{0}^{t} e(\sigma) d \sigma\right)
$$

where $K, T_{D}$, and $T_{I}$ are gains. The gains are selected as $K=1000, T_{D}=0.05$, and $T_{I}=0.2$. AFC is implemented with the PID. Parameters of AFC are $\eta=0.001$, and $\alpha=0.7$.

The TDCIVF, thanks to the impedance control formulation property, becomes a motion control formulation when the interaction torque $\boldsymbol{\tau}_{s}$ is omitted in the target dynamics (9). The motion control formulation for $1 \mathrm{DOF}$ is

$$
\tau=\tau_{t-L}-\bar{M} \ddot{\theta}_{t-L}+\bar{M}\left[\ddot{\theta}_{d}+K_{V}\left(\dot{\theta}_{d}-\dot{\theta}\right)+K_{p}\left(\theta_{d}-\theta\right)+\Gamma\left(\dot{\theta}_{\text {ideal }}-\dot{\theta}\right)\right]
$$

where

$$
\dot{\theta}_{\text {ideal }}=\int\left[\ddot{\theta}_{d}+K_{p}\left(\theta_{d}-\theta\right)+K_{V}\left(\dot{\theta}_{d}-\dot{\theta}\right)\right] d t .
$$

The desired error dynamics of TDCIVF is

$$
\ddot{\theta}_{d}-\ddot{\theta}+K_{V}\left(\dot{\theta}_{d}-\dot{\theta}\right)+K_{P}\left(\theta_{d}-\theta\right)=0
$$

or

$$
\ddot{\theta}_{d}-\ddot{\theta}+2 \zeta \omega_{d}\left(\dot{\theta}_{d}-\dot{\theta}\right)+\omega_{d}^{2}\left(\theta_{d}-\theta\right)=0
$$

with

$$
K_{V}=2 \zeta \omega_{d}, K_{P}=\omega_{d}^{2} .
$$

The gains of TDCIVF and the speed of the target error dynamics are selected as follows: $\bar{M}=0.1, \Gamma=50.0$, and $\omega_{d}=10$; and $\zeta=1$ for the fastest no overshoot performance. Shown in Fig. 12 (b), the tracking error of TDCIVF is the smallest, which confirms that the TDCIVF is superior to AFC. 
AFC attempts to adapt the polynomial coefficients of friction. The adaptation takes quite some sampling time to update the friction parameters due to its property of neural network (Visioli et al., 2001) that is normally slower than TDE as discussed in (Lee \& Oh, 1997). In contrast, the TDCIVF directly cancels most of nonlinearities using TDE and immediately activates the IVF when TDE is not sufficient. AFC must tune three gains of the PID by trial and error (it takes a quite some time to tune gains heuristically), and two additional parameters of AFC ( $\eta$ and $\alpha$ ). In the TDCIVF, after specifying the convergence speed of the error dynamics by selecting $\omega_{d}$ and $\zeta$, the tuning of the gains $\bar{M}$ and $\Gamma$ is systematic and straightforward as discussed in section 2.6. Consequently, the TDCIVF is an easier, more systematic, and more robust friction compensation method than AFC.

(a) Desired Trajectory
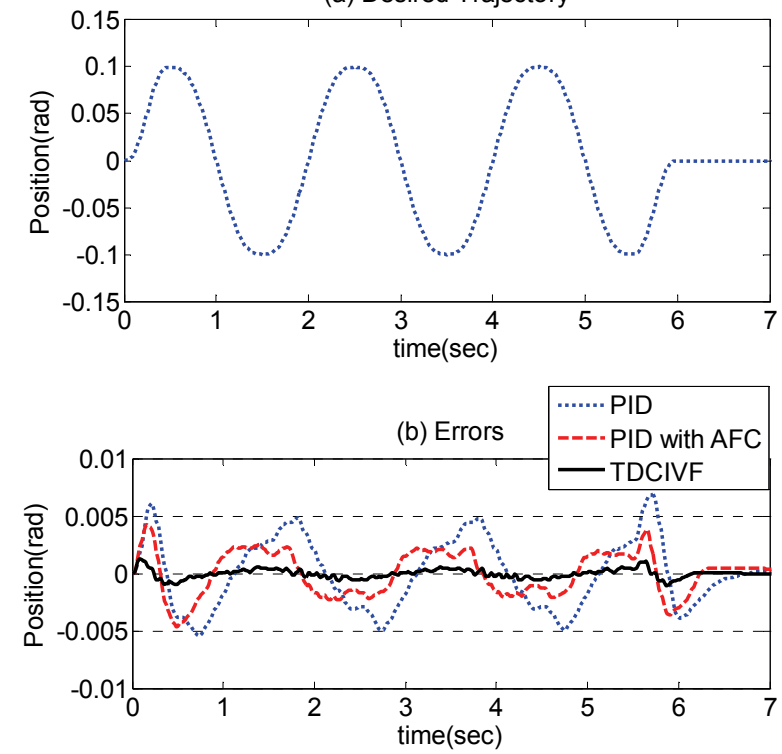

Figure 12. Experiment results on single arm with friction. (a) Desired trajectory.

(b) Comparison of tracking performance. (dotted: PID, dashed: PID with AFC, and solid: TDCIVF.)

\section{Cartesian Space Formulation}

The Cartesian state space equation of an n-DOF robot manipulator is given by

$$
\mathbf{F}_{u}=\mathbf{M}_{x}(\boldsymbol{\theta}) \ddot{\mathbf{x}}+\mathbf{V}_{x}(\boldsymbol{\theta}, \dot{\boldsymbol{\theta}})+\mathbf{G}_{x}(\boldsymbol{\theta})+\mathbf{F}_{x}(\boldsymbol{\theta}, \dot{\boldsymbol{\theta}})-\mathbf{F}_{s}
$$

where $\mathbf{F}_{u}$ denotes a fore-torque vector acting on the end-effector of the robot, $\mathbf{F}_{s}$ the interaction force, and $\mathbf{x}, \dot{\mathbf{x}}, \ddot{\mathbf{x}}$ is an appropriate Cartesian vector representing position and orientation of the end-effector; $\boldsymbol{\theta}, \dot{\boldsymbol{\theta}}, \ddot{\boldsymbol{\theta}} \in \mathfrak{R}^{n}$ denote the joint angle, the joint velocity, and the joint acceleration, respectively; $\mathbf{M}_{x}(\boldsymbol{\theta})$ is the Cartesian mass matrix; $\mathbf{V}_{x}(\boldsymbol{\theta}, \dot{\boldsymbol{\theta}})$ is a vector of 
velocity terms in Cartesian space, and $\mathbf{G}_{x}(\boldsymbol{\theta})$ is a vector of gravity terms in Cartesian space. $\mathbf{F}_{x}(\boldsymbol{\theta}, \dot{\boldsymbol{\theta}})$ is a vector of friction terms in Cartesian space (Craig, 1989). Note that the fictitious forces acting on the end-effector, $\mathbf{F}_{u}$, could in fact be applied by the actuators at the joints using the relationship

$$
\mathbf{\tau}=\mathbf{J}^{T}(\boldsymbol{\theta}) \mathbf{F}_{u}
$$

where $\mathbf{J}(\boldsymbol{\theta})$ is the Jacobian. The derivation of $\mathbf{M}_{x}(\boldsymbol{\theta}), \mathbf{V}_{x}(\boldsymbol{\theta}, \dot{\boldsymbol{\theta}}), \mathbf{G}_{x}(\boldsymbol{\theta}), \mathbf{F}_{x}(\boldsymbol{\theta}, \dot{\boldsymbol{\theta}})$ are given in (Craig, 1989), and expressed by

$$
\begin{gathered}
\mathbf{M}_{x}(\boldsymbol{\theta})=\mathbf{J}^{-T}(\boldsymbol{\theta}) \mathbf{M}(\boldsymbol{\theta}) \mathbf{J}^{-1}(\boldsymbol{\theta}) \\
\mathbf{V}_{x}(\boldsymbol{\theta}, \dot{\boldsymbol{\theta}})=\mathbf{J}^{-T}(\boldsymbol{\theta})\left(\mathbf{V}(\boldsymbol{\theta}, \dot{\boldsymbol{\theta}})-\mathbf{M}(\boldsymbol{\theta}) \mathbf{J}^{-1}(\boldsymbol{\theta}) \dot{\mathbf{J}}(\boldsymbol{\theta}) \dot{\boldsymbol{\theta}}\right) \\
\mathbf{G}_{x}(\boldsymbol{\theta})=\mathbf{J}^{-T}(\boldsymbol{\theta}) \mathbf{G}(\boldsymbol{\theta}) \\
\mathbf{F}_{x}(\boldsymbol{\theta}, \dot{\boldsymbol{\theta}})=\mathbf{J}^{-T}(\boldsymbol{\theta}) \mathbf{F}(\boldsymbol{\theta}, \dot{\boldsymbol{\theta}}) \\
\mathbf{F}_{x}(\boldsymbol{\theta}, \dot{\boldsymbol{\theta}})=\left(\mathbf{F}_{v}\right)_{x}+\left(\mathbf{F}_{c}\right)_{x}+\left(\mathbf{F}_{s t}\right)_{x}
\end{gathered}
$$

where $\left(\mathbf{F}_{v}\right)_{x},\left(\mathbf{F}_{c}\right)_{x}$, and $\left(\mathbf{F}_{s t}\right)_{x}$ denote viscous friction, Coulomb friction, and stiction terms in Cartesian space, respectively.

Introducing a matrix $\overline{\mathbf{M}}_{x}(\boldsymbol{\theta})$, another expression of (44) is obtained as follows:

$$
\mathbf{F}_{u}=\overline{\mathbf{M}}_{x}(\boldsymbol{\theta}) \ddot{\mathbf{x}}+\mathbf{N}_{x}(\boldsymbol{\theta}, \dot{\boldsymbol{\theta}}, \ddot{\boldsymbol{\theta}})
$$

where $\mathbf{N}_{x}(\boldsymbol{\theta}, \dot{\boldsymbol{\theta}}, \ddot{\boldsymbol{\theta}})$ and $\overline{\mathbf{M}}_{x}(\boldsymbol{\theta})$ are expressed by

$$
\begin{gathered}
\mathbf{N}_{x}(\boldsymbol{\theta}, \dot{\boldsymbol{\theta}}, \ddot{\boldsymbol{\theta}})=\left[\mathbf{M}_{x}(\boldsymbol{\theta})-\overline{\mathbf{M}}_{x}(\boldsymbol{\theta})\right] \ddot{\mathbf{x}}+\mathbf{V}_{x}(\boldsymbol{\theta}, \dot{\boldsymbol{\theta}})+\mathbf{G}_{x}(\boldsymbol{\theta})+\mathbf{F}_{x}(\boldsymbol{\theta}, \dot{\boldsymbol{\theta}})-\mathbf{F}_{s} \\
\overline{\mathbf{M}}_{x}(\boldsymbol{\theta}) \triangleq \mathbf{J}^{-T}(\boldsymbol{\theta}) \overline{\mathbf{M}} \mathbf{J}^{-1}(\boldsymbol{\theta})
\end{gathered}
$$

where $\overline{\mathbf{M}}$ is constant diagonal matrix.

The nonlinear term $\mathbf{N}_{x}(\boldsymbol{\theta}, \dot{\boldsymbol{\theta}}, \ddot{\boldsymbol{\theta}})$ can be classified into two categories: soft nonlinearities and hard nonlinearities, as follows:

$$
\begin{gathered}
\mathbf{N}_{x}(\boldsymbol{\theta}, \dot{\boldsymbol{\theta}}, \ddot{\boldsymbol{\theta}})=\mathbf{S}_{x}(\boldsymbol{\theta}, \dot{\boldsymbol{\theta}})+\mathbf{H}_{x}(\boldsymbol{\theta}, \dot{\boldsymbol{\theta}}, \ddot{\boldsymbol{\theta}}) \\
\mathbf{S}_{x}(\boldsymbol{\theta}, \dot{\boldsymbol{\theta}})=\mathbf{V}_{x}(\boldsymbol{\theta}, \dot{\boldsymbol{\theta}})+\mathbf{G}_{x}(\boldsymbol{\theta})+\left(\mathbf{F}_{v}\right)_{x}(\boldsymbol{\theta}, \dot{\boldsymbol{\theta}})-\mathbf{F}_{s} \\
\mathbf{H}_{x}(\boldsymbol{\theta}, \dot{\boldsymbol{\theta}}, \ddot{\boldsymbol{\theta}})=\left(\mathbf{F}_{c}\right)_{x}(\boldsymbol{\theta}, \dot{\boldsymbol{\theta}})+\left(\mathbf{F}_{s t}\right)_{x}(\boldsymbol{\theta}, \dot{\boldsymbol{\theta}})+\left[\mathbf{M}_{x}(\boldsymbol{\theta})-\overline{\mathbf{M}}_{x}(\boldsymbol{\theta})\right] \ddot{\mathbf{x}}
\end{gathered}
$$


The control objective in Cartesian space is to achieve following target impedance dynamics

$$
\mathbf{M}_{x d}\left(\ddot{\mathbf{x}}_{d}-\ddot{\mathbf{x}}\right)+\mathbf{B}_{x d}\left(\dot{\mathbf{x}}_{d}-\dot{\mathbf{x}}\right)+\mathbf{K}_{x d}\left(\mathbf{x}_{d}-\mathbf{x}\right)+\mathbf{F}_{s}=0
$$

where $\mathbf{F}_{s}$ denotes the interaction force; $\mathbf{M}_{x d}, \mathbf{B}_{x d}$, and $\mathbf{K}_{x d}$ denote the desired mass, the desired damping, and the desired stiffness in Cartesian space, respectively; and $\mathbf{x}_{d}, \dot{\mathbf{x}}_{d}, \ddot{\mathbf{x}}_{d}$ the desired position, the desired velocity, the desired acceleration in Cartesian space, respectively; $\mathbf{x}, \dot{\mathbf{x}}, \ddot{\mathbf{x}}$ denote the position, the velocity, and the acceleration in Cartesian space, respectively.

The Cartesian formulation of TDCIVF can be derived as follows:

$$
\mathbf{F}_{u}=\overline{\mathbf{M}}_{x}(\boldsymbol{\theta}) \mathbf{u}_{x}+\hat{\mathbf{S}}_{x}(\boldsymbol{\theta}, \dot{\boldsymbol{\theta}})+\hat{\mathbf{H}}_{x}(\boldsymbol{\theta}, \dot{\boldsymbol{\theta}}, \ddot{\boldsymbol{\theta}})
$$

where

$$
\mathbf{u}_{x}=\ddot{\mathbf{x}}_{d}+\mathbf{M}_{x d}^{-1}\left[\mathbf{K}_{x d}\left(\mathbf{x}_{d}-\mathbf{x}\right)+\mathbf{B}_{x d}\left(\dot{\mathbf{x}}_{d}-\dot{\mathbf{x}}\right)+\mathbf{F}_{s}\right]
$$

The estimation of $\hat{\mathbf{S}}_{x}(\boldsymbol{\theta}, \dot{\boldsymbol{\theta}})+\hat{\mathbf{H}}_{x}(\boldsymbol{\theta}, \dot{\boldsymbol{\theta}}, \ddot{\boldsymbol{\theta}})$ is given by

$$
\hat{\mathbf{S}}_{x}(\boldsymbol{\theta}, \dot{\boldsymbol{\theta}})+\hat{\mathbf{H}}_{x}(\boldsymbol{\theta}, \dot{\boldsymbol{\theta}}, \ddot{\boldsymbol{\theta}})=\mathbf{S}_{x}(\boldsymbol{\theta}, \dot{\boldsymbol{\theta}})_{t-L}+\mathbf{H}_{x}(\boldsymbol{\theta}, \dot{\boldsymbol{\theta}}, \ddot{\boldsymbol{\theta}})_{t-L}
$$

and

$$
\mathbf{S}_{x}(\boldsymbol{\theta}, \dot{\boldsymbol{\theta}})_{t-L}+\mathbf{H}_{x}(\boldsymbol{\theta}, \dot{\boldsymbol{\theta}}, \ddot{\boldsymbol{\theta}})_{t-L}=\left(\mathbf{F}_{u}\right)_{t-L}-\overline{\mathbf{M}}_{x}(\boldsymbol{\theta}) \mathbf{x}_{t-L} .
$$

Here, TDE error $\varepsilon$ is defined as

$$
\boldsymbol{\varepsilon} \triangleq \overline{\mathbf{M}}_{x}^{-1}(\boldsymbol{\theta})\left[\mathbf{H}_{x}(\boldsymbol{\theta}, \dot{\boldsymbol{\theta}}, \ddot{\boldsymbol{\theta}})-\mathbf{H}_{x}(\boldsymbol{\theta}, \dot{\boldsymbol{\theta}}, \ddot{\boldsymbol{\theta}})_{t-L}\right]
$$

Then, with the combination of (44), (54), (58), (59), (60), (62), impedance error dynamics is

$$
\mathbf{M}_{x d}\left(\ddot{\mathbf{x}}_{d}-\ddot{\mathbf{x}}\right)+\mathbf{B}_{x d}\left(\dot{\mathbf{x}}_{d}-\dot{\mathbf{x}}\right)+\mathbf{K}_{x d}\left(\mathbf{x}_{d}-\mathbf{x}\right)+\mathbf{F}_{s}=\mathbf{M}_{x d} \boldsymbol{\varepsilon} .
$$

$\varepsilon$ causes the resulting dynamics to deviate from the target impedance dynamics. To suppress $\varepsilon$, ideal velocity feedback term is introduced, with $\dot{\mathbf{x}}_{\text {ideal }}$ defined here as

$$
\dot{\mathbf{x}}_{i d e a l} \triangleq \int\left\{\ddot{\mathbf{x}}_{d}+\mathbf{M}_{x d}^{-1}\left[\mathbf{K}_{x d}\left(\mathbf{x}_{d}-\mathbf{x}\right)+\mathbf{B}_{x d}\left(\dot{\mathbf{x}}_{d}-\dot{\mathbf{x}}\right)+\mathbf{F}_{s}\right]\right\} d t .
$$

Combining previous formulations, the control law is

$$
\begin{aligned}
\mathbf{F}_{u}= & \left(\mathbf{F}_{u}\right)_{t-L}-\overline{\mathbf{M}}_{x}(\boldsymbol{\theta}) \mathbf{x}_{t-L} \\
& +\overline{\mathbf{M}}_{x}(\boldsymbol{\theta})\left\{\ddot{\mathbf{x}}_{d}+\mathbf{M}_{x d}^{-1}\left[\mathbf{K}_{x d}\left(\mathbf{x}_{d}-\mathbf{x}\right)+\mathbf{B}_{x d}\left(\dot{\mathbf{x}}_{d}-\dot{\mathbf{x}}\right)+\mathbf{F}_{s}\right]\right\} \\
& +\overline{\mathbf{M}}_{x}(\boldsymbol{\theta}) \boldsymbol{\Gamma}\left(\dot{\mathbf{x}}_{i d e a l}-\dot{\mathbf{x}}\right)
\end{aligned}
$$

where $\Gamma$ is a gain matrix. 


\section{Human-Robot Cooperation}

The experiment scenario for a human-robot cooperation task is as follows: The robot endeffector draws a circle in $4 \mathrm{~s}$ in free space and afterwards stands still before it is pushed by a human. A human pushes the robot end-effector forth and back in $Y$ direction while the robot tries to keep in contact with it. This is to simulate the situation, for example, when a worker and a robot work together to move or install an object. In this cooperation task, it is often desired for the robot end-effector to behave like a low-stiffness spring, and the target dynamics is described as follow:

$$
\mathbf{M}_{x d}=\left[\begin{array}{cc}
20 & 0 \\
0 & 20
\end{array}\right] \mathrm{Kg} \text { and } \mathbf{K}_{x d}=\left[\begin{array}{cc}
2000 & 0 \\
0 & 500
\end{array}\right] \mathrm{N} / \mathrm{m} .
$$

$\zeta=1.0$ for free space motion and $\zeta=6.0$ for constrained space. The $\mathrm{Y}$ direction stiffness of the target impedance is low $(500 \mathrm{~N} / \mathrm{m})$ for the compliant interaction with human.

Experimental results are displayed in Fig. 8 and Fig. 9. The end-effector of the robot acts like a soft spring-mass-damper system to a human being. It neither destabilizes the robot nor exerts an excessive force to the human being. In free space task, shown in Fig. 10 (b), the TDCIVF is better than IFBIC at tracking the circle. In the robot-human cooperation task, shown in Fig. 10 (d), the TDCIVF shows the smallest impedance error.

This experiment illustrates that human-robot cooperative tasks can be accomplished successfully under TDCIVF. The TDCIVF shows robust compliant motion control while possessing soft compliance.

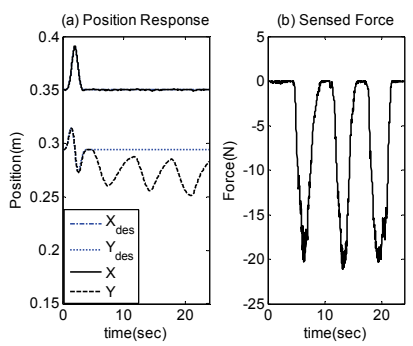

Figure 8. Human-Robot Cooperation: Position and force responses of IFBIC

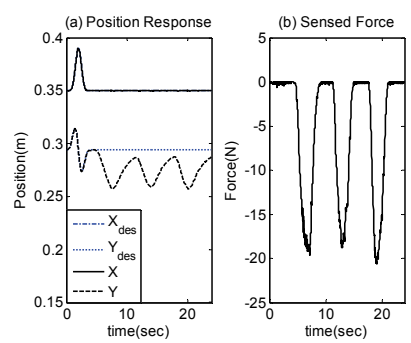

Figure 9. Human-Robot Cooperation: Position and force responses of TDCIVF 
(a) IFBIC

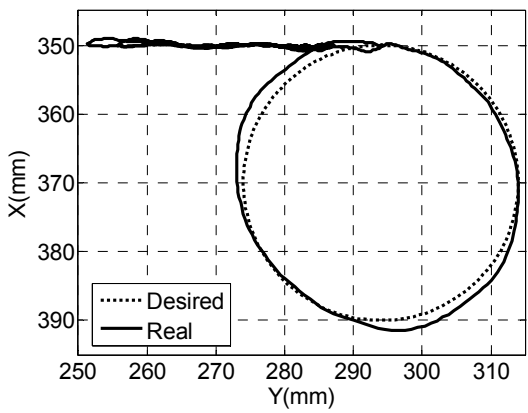

(c) Human-Robot Physical Interaction

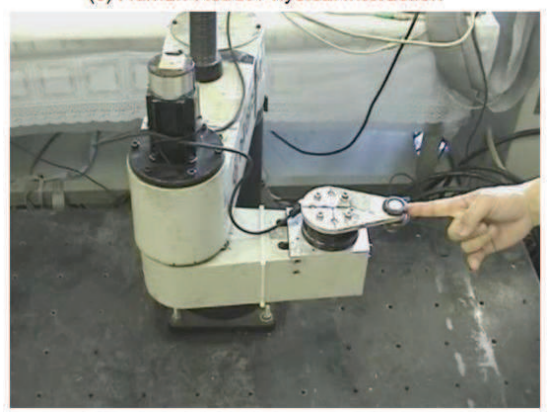

(b) TDCIVF

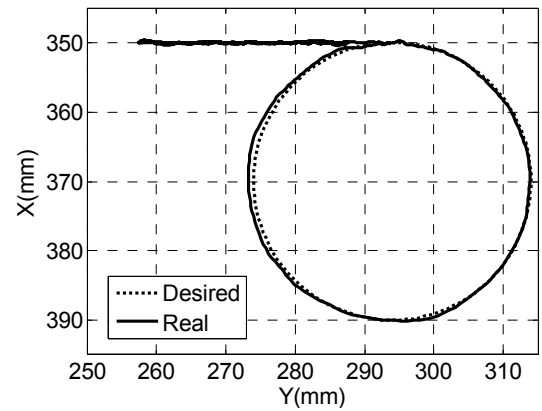

(d) Impedance Error

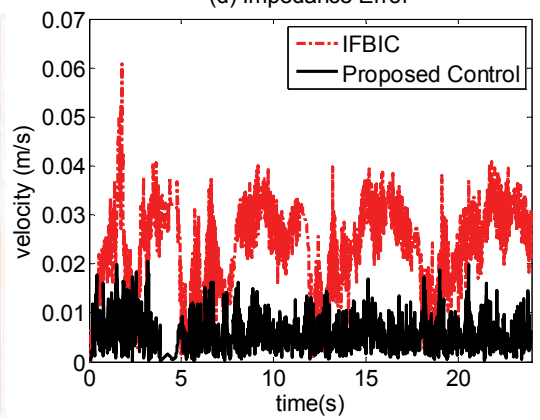

Figure 10. Human-Robot Cooperation. (a) IFBIC: only TDE is used. (b) TDCIVF: both TDE and IVF is used. (c) Experiment scienario. (d) Comparison of impedance error

\section{Conclusion}

The TDCIVF has following properties:

1. Transparent control structure.

2. Simplicity of gain tuning.

3. Non-model based online friction compensation.

4. Robustness against both soft nonlinearities and hard nonlinearities.

The overall implementation procedure of TDCIVF is straightforward and practical. The cancellation effect of soft nonlinearities using TDE and the suppression effect of hard nonlinearities using ideal velocity feedback are confirmed through simulation studies. The TDCIVF can reduce the effect of hard nonlinearities by raising the gain $\Gamma$.

Compared with AFC, a recently developed non-model based method, the TDCIVF provides a more systematic, easier, and more robust friction compensation method. The human-robot cooperation experiment shows that the TDCIVF is practical for realizing compliant manipulation of robots.

The following directions can be considered for future research:

1. The term caused by hard nonlinearity, $\varepsilon$ in (16) may be regarded as perturbation to the target impedance dynamics. It is noteworthy for further research that the TDE error $\varepsilon$ can be suppressed by other compensation methods. 
2. The terms in robot dynamics considered in this TDCIVF are: the gravity term, viscous friction term, the Coriolis and centrifugal term, the disturbance term, and the environmental force term (soft nonlinearities); and the Coulomb friction term, the stiction term, and the inertia uncertainty term (hard nonlinearities). From the TDE viewpoint, consideration and compensation of other terms such as saturation, backlash and hysteresis terms can be investigated in depth.

3. Without modelling robot dynamics and nonlinear friction, the TDCIVF provides robust compensation and fast accurate trajectory tracking. Recently, the Lugre friction mode based control has attracted research activities because the Lugre model is known as accurate friction model in friction compensation literature. It will be interesting to experimentally compare the TDCIVF (which uses no friction model) with the Lugre friction model based control.

\section{References}

Aghili F. \& Namvar M. (2006). Adaptive control of manipulators using uncalibrated jointtorque sensing, IEEE Transactions on Robotics, Vol. 22, No. 4, pp. 854-860.

Armstrong-Helouvry B.; Dupont P. \& de Wit C. C. (1994). A survey of models, analysis tools and compensation methods for the control, Automatica, Vol. 30, No. 1, pp. 10831138.

Bona B. \& Indri M. (2005). Friction compensation in robotics: An overview, Proceedings of 44th IEEE International Conference on Decision and Control and 2005 European Control Conference (CDC2005), pp. 4360- 4367.

Bona B.; Indri M. \&, Smaldone N. (2006). Rapid prototyping of a model-based control with friction compensation for a direct-drive robot, IEEE-ASME Transactions on Mechatronics, Vol. 11, No. 5, pp. 576-584.

Bonitz R. C. \& Hsia T. C. (1996). Internal force-based impedance control for cooperating manipulators, IEEE Transactions on Robotics and Automation, Vol. 12, No. 1, pp. 7889.

Craig J.J. (1989). Introduction to Robotics: Mechnaics and Control, Addison-Wesley, ISBN-13: 9780201095289.

Hsia T. C.; Lasky T. A. \& Guo Z. (1991). Robust independent joint controller design for industrial robot manipulators, IEEE Transactions on Industrial Electronics, Vol. 38, No. 1, pp. 21-25.

Jatta F.; Legnani G. \& Visioli A. (2006). Friction compensation in hybrid force/velocity control of industrial manipulators, IEEE Transactions on Industrial Electronics, Vol. 53, No. 2, pp. 604-613.

Jin M.; Kang S. H. \& Chang P. H. (2006). A robust compliant motion control of robot with certain hard nonlinearities using time delay estimation. Proceedings - IEEE International Symposium on Industrial Electronics (ISIE2006), Montreal, Quebec, Canada, pp. 311-316.

Jin M.; Kang S. H. \& Chang P. H. (2008). Robust compliant motion control of robot with nonlinear friction using time delay estimation. IEEE Transactions on Industrial Electronics, Vol. 55, No. 1, pp. 258-269.

Lasky T. A. \& Hsia T. C. (1991). On force-tracking impedance control of robot manipulators, Proceedings - IEEE International Conference on Robotics and Automation (ICRA'91), pp. 274-280. 
Lee J.-W. \& J.-H. Oh (1997). Time delay control of nonlinear systems with neural network modelling, Mechatronics, Vol. 7, No. 7, pp. 613-640.

Lischinsky P.; de Wit C. C. \& Morel G. (1999). Friction compensation for an industrial hydraulic robot, IEEE Control System Magazine, Vol 19, No. 1, pp. 25-32.

Liu G.; Goldenberg A. A. \& Zhang Y. (2004), Precise slow motion control of a direct-drive robot arm with velocity estimation and friction compensation, Mechatronics, Vol. 14, No. 7, pp. 821-834.

Marton L. \& Lantos B. (2007). Modeling, identification, and compensation of stick-slip friction, IEEE Transactions on Industrial Electronics, Vol. 54, No. 1, pp. 511-521.

Mei Z.-Q.; Xue Y.-C. \& Yang R.-Q. (2006). Nonlinear friction compensation in mechatronic servo systems, International Journal of Advanced Manufacturing Technology, Vol. 30, No. 7-8, pp. 693-699.

Slotine J.-J. E. (1985). Robustness issues in robot control, Proceedings - IEEE International Conference on Robotics and Automation (ICRA'1985), Vol. 3, pp. 656-661.

Xie W.-F. (2007). Sliding-mode-observer-based adaptive control for servo actuator with friction, IEEE Transactions on Industrial Electronics, Vol. 54, No. 3, pp. 1517-1527.

Visioli A.; Adamini R. \& Legnani G. (2001). Adaptive friction compensation for industrial robot control, Proceedings-IEEE/ASME International Conference on Advanced Intelligent Mechatronics (AIM2001), Vol. 1, Jul. 2001, pp. 577-582.

Visioli A.; Ziliani G. \& Legnani G. (2006). Friction compensation in hybrid force/velocity control for contour tracking tasks, In: Industrial Robotics: Theory, Modeling and Control, Sam Cubero (Ed.), pp. 875-894. Pro Literatur Verlag, Germany / ARS, Austria, ISBN 3-86611-285-8.

Youcef-Toumi K. \& Ito O. (1990). A time delay controller design for systems with unknown dynamics, Journal of Dynamic Systems Measurement and Control-Transactions of the ASME, Vol. 112, No. 1, pp. 133-142. 


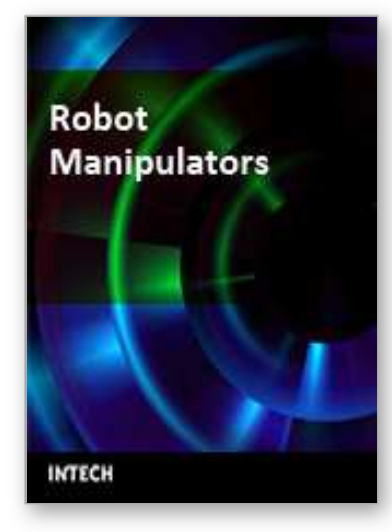

\author{
Robot Manipulators \\ Edited by Marco Ceccarelli
}

ISBN 978-953-7619-06-0

Hard cover, 546 pages

Publisher InTech

Published online 01, September, 2008

Published in print edition September, 2008

In this book we have grouped contributions in 28 chapters from several authors all around the world on the several aspects and challenges of research and applications of robots with the aim to show the recent advances and problems that still need to be considered for future improvements of robot success in worldwide frames. Each chapter addresses a specific area of modeling, design, and application of robots but with an eye to give an integrated view of what make a robot a unique modern system for many different uses and future potential applications. Main attention has been focused on design issues as thought challenging for improving capabilities and further possibilities of robots for new and old applications, as seen from today technologies and research programs. Thus, great attention has been addressed to control aspects that are strongly evolving also as function of the improvements in robot modeling, sensors, servo-power systems, and informatics. But even other aspects are considered as of fundamental challenge both in design and use of robots with improved performance and capabilities, like for example kinematic design, dynamics, vision integration.

\title{
How to reference
}

In order to correctly reference this scholarly work, feel free to copy and paste the following:

Maolin Jin, Sang Hoon Kang and Pyung Hun Chang (2008). Simple Effective Control for Robot Manipulators with Friction, Robot Manipulators, Marco Ceccarelli (Ed.), ISBN: 978-953-7619-06-0, InTech, Available from: http://www.intechopen.com/books/robot_manipulators/simple_effective_control_for_robot_manipulators_with_f riction

\section{INTECH}

open science | open minds

\author{
InTech Europe \\ University Campus STeP Ri \\ Slavka Krautzeka 83/A \\ 51000 Rijeka, Croatia \\ Phone: +385 (51) 770447 \\ Fax: +385 (51) 686166 \\ www.intechopen.com
}

\author{
InTech China \\ Unit 405, Office Block, Hotel Equatorial Shanghai \\ No.65, Yan An Road (West), Shanghai, 200040, China \\ 中国上海市延安西路65号上海国际贵都大饭店办公楼405单元 \\ Phone: +86-21-62489820 \\ Fax: +86-21-62489821
}


(C) 2008 The Author(s). Licensee IntechOpen. This chapter is distributed under the terms of the Creative Commons Attribution-NonCommercialShareAlike-3.0 License, which permits use, distribution and reproduction for non-commercial purposes, provided the original is properly cited and derivative works building on this content are distributed under the same license. 